\title{
Challenges in the governance of biomedical and health research after publication of the New Drugs and Clinical Trial Rules, 2019
}

\author{
YASHASHRI SHETTY, VISHAL KRIPASHANKAR SINGH
}

\begin{abstract}
This article pertains to the governance of biomedical and health research after the publication of the New Drugs and Clinical Trial Rules, 2019. The new regulations cover all types of research. However, those concerning biomedical and health research may fall short on certain counts regarding: reviewing studies with vulnerable populations, undertaking risk benefit analysis, finding relatedness in case of serious adverse events, and providing compensation. The challenges faced by ethics committees from the inception of a study to its completion are discussed. There is a need for rigorous training of all members involved in the ethics committee's functioning.
\end{abstract}

\section{Introduction}

The Ministry of Health and Family Welfare (MoHFW), Government of India, notified the New Drugs and Clinical Trials Rules, 2019, in March 2019 (1). The new rules superseded Part XA and Schedule $Y$ of the Drugs and Cosmetics Rules with immediate effect. The New Rules are particularly important because they extend beyond governance of clinical trials conducted towards marketing approval to what is described as biomedical and health research (BMHR).

The New Drugs and Clinical Trials Rules, 2019 define BMHR as research including studies on basic, applied and operational research or clinical research, designed primarily to increase scientific knowledge about diseases and conditions (physical or socio-behavioral); their detection and cause; and evolving strategies for health promotion, prevention, or amelioration of disease and rehabilitation but does not include clinical trial...;

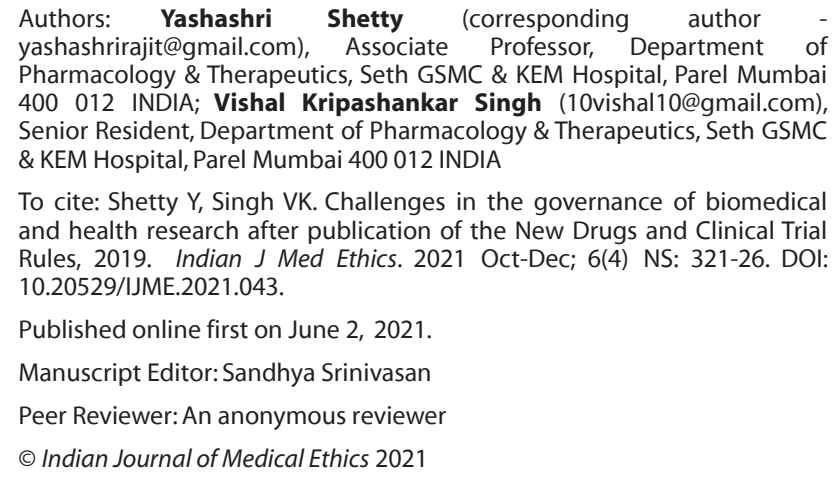

To cite: Shetty $Y$, Singh VK. Challenges in the governance of biomedical and health research after publication of the New Drugs and Clinical Trial Rules, 2019. Indian J Med Ethics. 2021 Oct-Dec; 6(4) NS: 321-26. DOI: 10.20529/IJME.2021.043.

Published online first on June 2, 2021.

Manuscript Editor: Sandhya Srinivasan

Peer Reviewer: An anonymous reviewer

c Indian Journal of Medical Ethics 2021

The Rules define a clinical trial (CT) as

any systematic study of such new drug or investigational new drug in human subjects to generate data for discovering or verifying its, -

i. clinical;

ii. or pharmacological including pharmacodynamics, pharmacokinetics or;

iii. adverse effects,

with the objective of determining the safety, efficacy or tolerance of such new drug or investigational new drug; (1)

All clinical trials undertaken with marketing intent are considered regulatory studies. This includes studies testing drugs, biologicals, devices, phytopharmaceuticals, stem cells, and cosmetics. Non-regulatory studies, including studies with academic intent, may be reviewed by BMHR ECs.

There will be two ethics committees (EC) to cater to these studies:

- The Clinical Trial Ethics Committee (CT EC), constituted under Rule 7 and registered under Rule 8, governed by the Central Drugs Standard Organization-CDSCO [Ethics Committee Registration Division] (2), and

- The Biomedical and Health Research Ethics Committee (BMHR EC), constituted under Rule 16 and registered under Rule 17, governed by the Department of Health Research (DHR), Ministry of Health and Family Welfare (MoHFW), Government of India, and National Ethics Committee Registry for Biomedical and Health Research (3)

Institutions - government, private or non-governmental conducting clinical trials are required to follow the CT Rules of 2019. Any institution - government, private or nongovernmental - wishing to undertake BMHR must follow the National Ethical Guidelines for Biomedical and Health Research Involving Human Participants, 2017 (4).

\section{The ICMR's ethical guidelines, 2017}

The ICMR's ethical guidelines for biomedical and health research involving human participants of 2017 contain a number of additions or refinements to the 2006 guidelines. 
First, the description of "vulnerable populations", defined in the 2006 guidelines as "economically or socially disadvantaged groups", has been expanded. Section 6 of the 2017 guidelines mentions specific economic and socially disadvantaged groups - unemployed individuals, orphans, abandoned individuals, persons below the poverty line, ethnic minorities, and sexual minorities including lesbian/gay/ bisexual and transgender (LGBT). When research is planned on vulnerable persons, it is the EC's and the investigator's responsibility to protect their interests because they cannot themselves do so, or are in a compromised position that makes them unable to protect their interests on their own.

Second, new sections have been added. Public health research and social and behavioural science research were combined in the 2006 guidelines but in the 2017 guidelines these have been discussed in separate sections. Guidelines on research during humanitarian emergencies and disasters have been added as a new section (Section 12); this includes both man-made and natural disasters. Guidelines for research on biological materials, biobanking, and datasets (Section 11) have also been added.

Third, the 2017 guidelines describe specific types of consent applicable to different types of studies. One such consent is "broad consent". For example, in an academic setting, if investigators want to store samples prospectively for some years for future research, a participant's broad consent allows for current and future access, and use of samples or data for research. There is no need to approach the participant again, if the researcher wants to do research on her/his sample in future.

Another specific type of consent is "reconsent". In the case of children enrolled in longitudinal studies, when the child becomes an adult, investigators must "reconsent" the now adult participant. The guidelines also refer to online consent in research involving sensitive data, such as studies on high risk behaviour or in vaccine research; partner or spouse consent as additional consent required in a genetic study if information about the secondary participants is identifiable; and consent in studies that involve deception. In the last case, informed consent is not possible due to the nature of research. Here, a two-step procedure may be required, comprising an initial consent and a second request for consent following a debriefing after participation.

Fourth, the 2017 guidelines have made provision to review multicentric research by a single EC. For multicentric biomedical and health research, all participating sites may decide to utilise the services of one common EC from a participating site that is identified as the designated main EC for the purpose of primary review. However, the local site requirements, such as monitoring of the informed consent process and of research implementation, may be performed by the local EC (4).

These sections were missing or not given in such detail in the 2006 guidelines.

\section{Registration of ECs}

Institutions desirous of conducting CTs or BMHR are required to get registration from the specified authorities as provided in Rule 8 and Rule 17 respectively. Ethics review and clearance may be given by institutional or independent ECs. For registration of institutional and independent ECs, a checklist of documents to be uploaded is provided on the Department of Health Research website (for BMHR) and in the New Drugs and CT Rules, 2019 (for CTs). The checklist includes:

- an application for registration with a signed copy of the covering letter,

- a letter of authority under which the ethics committee has been constituted,

- the membership requirements of the ethics committee, with the terms of reference,

- the conditions of appointment to the EC, and the quorum required (with the relevant SOPs),

- the list of EC members with proof of their qualifications (detailed curriculum vitae, updated degree certificates and training certificates in ethics and GCP),

- the ECs policies for dealing with conflict of interest, for reviewing protocols involving research on vulnerable populations, and for training of new members,

- documents showing whether the committee has been audited, and

- an undertaking by the committee with Form CT-01.

Both CT ECs and BMHR ECs have the same documentation list. Once the documents are uploaded, the EC receives a query or a note of approval. CDSCO registration started in 2017 under Rule 122DD and was initially given for three years. At present, it is carrying out re-registration of ECs reviewing regulatory studies, and is granting approval for five years. The DHR which registers ECs reviewing BMHR gives provisional registration for two years, during which it will scrutinise the documents and, if satisfied with them, issue final registration for five years. If the DHR is not satisfied with the applicant, it can reject the application by providing reasons.

Registration of ECs is a good thing; it is necessary to know the number of ECs functioning in the country, and to confirm that their functioning, and the contribution of every member in the meetings, meets certain standards.

There are 1,570 ECs re-registered on the CDSCO website as on May 21, 2021; of these, 1,412 are institutional and 158 are independent ECs. Registration on the DHR website started in September 2019 (1,3).

The New Drugs and Clinical Trials Rules, 2019, for BMHR ECs do not mention the monitoring of registered ECs. So, they will only be reviewed by passive monitoring when the 
documentation is provided during the re-registration.

\section{The role and responsibilities of ethics committees}

All ECs are supposed to review protocols in detail for their scientific correctness (the objectives, recruitment procedure, design, and methodology), ethical issues (whether or not the populations involved are vulnerable, whether risk-benefit analysis has been conducted, privacy and confidentiality maintained, and informed consent taken), and legal compliance (whether the country guidelines are followed, appropriate permissions and MoUs of collaborators are in place, the trial is insured, the clinical trial agreement is adequate, and the investigator undertaking is in place). (4)

Public health research involves data collection through surveillance, vital statistics, disease reporting and registries; investigation of outbreaks, monitoring of the use of preventive interventions and health promotion; monitoring and programme evaluation; and enforcing of mandatory requirements, such as screening, treatment, immunisation, notifying diseases and, depending on the situation, quarantine. Social and behavioural science studies include anthropology, sociology, psychology, philosophy, political science, history, economics, communications and education.

The types of studies reviewed by a BMHR EC would pertain to trials of modern medicine for approved drugs, devices or biological interventions (as compared to a CT EC which will review trials of new drugs for regulatory approval); trials of traditional systems of medicine, trials of devices, diagnostics and surgical interventions, questionnaire-based studies, and death certificate analysis.

\section{Risk and harm in BMHR}

As stated in the ICMR 2017 guidelines, the type of EC review (full board review, expedited review and exemption from review) is based on the risk involved in the research, compared to the minimal risk which is the probability and magnitude of harms ordinarily encountered in daily life. Risk can be less than minimal (eg, research on anonymised or nonidentified data/samples), minimal (eg, observational studies), a minor increase over minimal risk, or low risk (eg, use of personal identifiable data in research, which can impose social risks, psychological harm, and economic harm), and more than minimal risk or high risk (eg, intervention in vulnerable populations).

Researchers, funding agencies, and EC members must be trained to categorise studies as per the risk categories mentioned in the ICMR guidelines.

While a minimal risk protocol will not require too many safeguards, high-risk protocols will require safeguards including withdrawal criteria and stopping rules for the study, establishing a Data Safety and Monitoring Board (DSMB), and possibly setting up safeguards to protect the confidentiality of sensitive personal data. For example, if an academic clinical trial is based on the design of a randomised controlled trial to evaluate the efficacy of a changed dose of an approved drug to the approved drug itself, the EC can ask for stopping rules and appoint an institutional DSMB to monitor the study progress.

BMHR studies judged to pose less than minimal risk (using data in the public domain) may be exempt from ethics review; those with minimal risk (research on non-identifiable specimens) may undergo expedited review. Research during emergencies and disasters may get expedited review with post-study review as well. All research proposals presenting more than minimal risk must undergo full committee review. This includes research involving vulnerable populations, studies involving the deception of participants, and research on predictable emergencies. This review decision is taken on a case-to-case basis.

While in clinical trials, harm is understood as physical harm from the drugs or devices being studied, the concept of harm is more complicated in studies which fall under BMHR, where there is often insufficient awareness of non-physical types of harm. Moreover, in a BMHR study, the balance of benefit and risk to individuals and to society will depend on the local values of the society.

Harm in a BMHR study could be social (stigma and discrimination), economic (loss of work) or psychological (interviewing on sensitive issues). Hence confidentiality must be maintained during and after the study, and the resulting data must be published in a manner that is respectful of the interests of all concerned. Questionnaire-based studies should be scrutinised beforehand, and questions reframed if needed. Database studies must be reviewed for the mechanisms to maintain privacy and confidentiality of data. A review of studies using biological samples may look at the type of consent required for potential future use of the samples.

Studies that fall in the category of BMHR sometimes use deception as part of the study design. The notion of deception is counter to research ethics, and ECs must approve studies with deception only in exceptional situations. For this, members should be trained to understand the use of deception-why it is used, and under what circumstances it might be permitted. Deception is not permissible in cases in which the study exposes participants to more than minimal risk.

\section{Written consent in BMHR}

Researchers should obtain written consent wherever appropriate. Regarding community research, if the community is not comfortable with giving written consent, the EC may give consent waivers if the study falls below minimal risk. The guidelines state that when written consent is not feasible, verbal consent is acceptable provided that it is formally documented and witnessed. However, this can sometimes be misused. Likewise, waiver of consent can be misused, a well-known example being that of the human 
papilloma virus (HPV) vaccine study on children which took the consent of hostel wardens and not of the parents. The researchers argued that the requirements of a clinical trial did not apply as this was a "demonstration study." (5)

\section{Challenges faced by an ethics committee when reviewing BMHR}

\section{Shortage of independent members}

The New Drugs and Clinical Trials Rules, 2019, have defined the composition of the EC according to the ICMR guidelines 2017. Fifty percent of the members should be non-affiliated, the number of members in an EC should preferably be between seven and 15 , and a minimum of five members should be present to meet quorum requirements. (4) These rules can ensure that the EC's decision is not biased by the interests of institutional members.

However, it is difficult for ECs situated where there is a single medical college to get non-affiliated members. Getting nonaffiliated members from another city entails additional expense for the EC's functioning.

\section{Shortage of members with the necessary expertise}

When BMHR studies are conducted at institutes dedicated to social and behavioural health research, the BMHR EC is likely to have the expertise to ensure a robust review process. However, proposals for research that fall in the category of BMHR are submitted to many medical colleges; such studies may have been conducted earlier, but started getting reviewed only after the New Rules came into effect.

As investigators doing research in social science or public health are not necessarily trained to write a fool-proof protocol, they may not define risk and harm in the protocol or in the informed consent document. When the EC that is reviewing such research does not have the expertise to identify such problems, the study may be judged to be of less than minimum risk and the EC may exempt it from review, or grant expedited review.

For this reason BMHR ECs must appoint experts in social and behavioural health research. However, such experts are scarce, and getting such experts is a challenge for the ECs.

\section{Need for adequate guidance on specialised research}

Neither the ICMR 2017 guidelines nor the New Clinical Trials Rules give guidance for the review of studies on artificial intelligence ( $\mathrm{Al})$, which is rapidly entering the fields of diagnostics, therapeutics, screening, and surgery. Some of the ethical issues in Al studies are similar to those in dataset studies. Al depends on digital data, and inconsistencies in the availability and quality of data restrict the potential of Al. Physician and patient acceptance is also an issue. Al protocols are at a primitive stage and replacing humans in diagnostics pharmacovigilance or in monitoring seems impractical. Without guidance on this subject, different ECs may give conflicting decisions on such studies.

Complementary medicine (except the traditional system of medicine) has not been represented in the guidelines. For Ayurveda, Yoga and Naturopathy, Unani, Siddha and Homeopathy (AYUSH), it is mentioned that the same ethical principles should be followed but no guidance is provided.

\section{Ensuring financial responsibilities are met in BMHR}

Some investigator-initiated or academic studies can be trials of changes in dose, dosage form, duration or indication for an approved drug. Such trials can pose more than minimal risk to the participants, and the trial's budget must include financial coverage for payment for participation and compensation for study-related injury. The principal investigator (PI) is responsible for the totality of research, as the sponsor would have in regulatory trials, and must make budgetary arrangements for the conduct of the study, including investigations and any intervention-related treatment. The PI must also provide insurance or give an undertaking that $s / h e$ is ready to pay compensation if any serious adverse event (SAE) occurs that is related to the trial. As per the ICMR guidelines, the host institution is also responsible for compensation or insurance coverage for research-related (physical) injury and harm (4). The causality assessment and calculation for compensation done by the EC is the same as that done for clinical trials.

However, such arrangements are often missing. There are instances of the EC taking a decision based on the PI's undertaking, without any financial arrangement in place. In the absence of clarity on this issue, some ECs have allowed such studies, but others have not. With the New Drugs and Clinical Trials Rules, 2019, ECs will have to be vigilant and ensure that principal investigators (PIs) and host institutions have made budgetary arrangements to cover all expenses of the study, including compensation; if something goes wrong in such research, ECs can also face legal action.

\section{Untrained investigators in academic studies}

In our experience, the protocol of an intervention study submitted by investigators is often incomplete in at least one of the components - the rationale, the study design, the description of the methodology, sample size, and statistical tests. Sometimes, the investigator does not define harm in the protocol, or perform a risk-benefit analysis. The definition of a vulnerable population and why it is vulnerable are not always defined in the protocol. One possible reason for this is that investigators are not trained properly in the conduct of research. Some investigators are resident doctors doing research for the first time for their dissertations. The workload of an EC increases because the review process has to be extensive in these cases. If the documentation is weak, the EC will be forced to raise many queries, and there will be a delay in the approval process. Sometimes investigators do not have the training necessary to address the EC's queries, and give up on the proposal (6). This is a waste of the EC's time. 
Furthermore, if the EC members are untrained, they may give approval to an inadequate proposal without proper ethics review.

\section{Challenges in collaborative research.}

Some research for drugs or diagnostics under BMHR is a collaboration between institutions and sponsors. Sponsors can be individuals, private companies, non-governmental organisations or specialty trusts. The sponsor may pay for drugs or investigations for the study and take samples of unwanted tissue to conduct secondary research, store it for future research, or use it to create products. Before the New Rules, funders who sponsored such research as a marketing tactic, or to get additional information that they could add to their drug label, could bypass ethics review. This is no longer possible under the New Rules, as such research would have to be reviewed by a BMHR EC.

Under the New Rules, the PI submitting such a proposal for EC review must also submit the memorandum of understanding (MoU) between the collaborating institutions or individuals doing such research. This MoU should describe the objectives of the collaboration, the rights of patients, and the roles and responsibilities of investigators and institutions. This information must also be contained in the Informed Consent Document.

However, such details are not always declared and presented in full in the documents submitted to the BMHR EC. Identifying lapses in the course of reviewing such protocols requires training and vigilance on the part of ECs.

Government-funded studies that fall under BMHR often do not mention payment for participation or compensation for study-related injury. The government may refuse to take the responsibility of a sponsor. This is a challenge for the EC. Moreover, in our experience, ECs tend to be biased towards government-funded studies, and approve such studies. The central government should take these issues into consideration while sanctioning funds for any type of interventional human research.

In research on traditional systems of medicine, insurance is not usually provided by the company doing research on the marketed product. Although the MoU mentions the roles and responsibilities of all collaborators and the management of free patient care, the risk of such research is often not defined as the medicines are multi-ingredient products, or because adequate literature is not available. Reviewing and approving such studies is challenging for the EC. The New Drugs and Clinical Trials Rules, 2019, do not give any guidance for reviewing such studies.

\section{Challenges faced during and after the conduct of the study}

Most ECs rely on passive monitoring to ensure the quality of the data generated and the safety of the intervention, in the form of annual updates and safety updates. However, we have found that the PI does not file the update unless reminded by the EC, and on occasion the EC forgets to send a reminder, deviating from its own SOPs.

Reports that are to be submitted annually are often incomplete. They may not mention the number of participants approved, the number screened, the number enrolled, whether any participants have withdrawn, and the reasons for this. If reports are not submitted annually, participants recruited in the interim period are not under EC oversight. It is difficult for an EC to take action against such PIs.

If the protocols of academic studies do not define harm, protocol deviations (7), adverse effects and serious adverse effects (8), these will not get reported by investigators. Without the power to conduct active monitoring of such studies, the EC must rely on the data and the risk-benefit analysis provided by the PI to decide whether or not to allow the study to continue. It is difficult for the EC to take such decisions when the data are incomplete.

Among the challenges common to proper oversight of CT and BMHR is that of humanpower and administration. Both ECs must fight for the resources to ensure that investigators submit study completion reports; both find it difficult to enforce the requirement that completion reports must be reviewed and approved before articles may be submitted for publication.

The requirement that the EC should maintain documents related to the clinical study for a period of five years after completion (1) adds to the burden of administration. ECs have always had issues with limited space, and now they need additional space for archives as per the New Drugs and Clinical Trials Rules, 2019.

Independent ECs reviewing academic studies conducted in private clinics or institutions face additional problems as they have no institutional funding.

Addressing such issues, and training of new and existing committee members on standard operating procedures (SOPs) as is required in the New Rules (1), will enhance the EC review process and bring uniformity across ECs in India.

\section{Conclusion}

The New Drugs and Clinical Trials Rules, 2019, contain provisions to fill gaps in the existing functioning of ECs reviewing health research other than regulatory clinical trials. This article on the challenges of reviewing BMHR highlights certain limitations in these provisions. If the Rules can be amended to address these flaws, there will be a significant improvement in the review of such research.

\section{References}

1. Ministry of Health and Family Welfare. Govt of India. Notification. The Gazette of India: Extraordinary, Part II, Section 3, Subsection (i), New Delhi, March 2019 . [cited 2019 Nov 1]. p. 1-264. Available from: https://cdsco.gov.in/opencms/export/sites/CDSCO_WEB/Pdfdocuments/NewDrugs_CTRules_2019.pdf 
2. Central Drugs Standard Control Organization: Ethics Committee ReRegistration [cited 2019 Nov 1]. Available from: https://cdsco.gov.in/ opencms/opencms/en/Clinical-Trial/Ethics-Committee/EthicsCommittee-Re-Registration/

3. Department of Health Research, Ministry of Health \& Family Welfare, Government of India. National Ethics Committee Registry for Biomedical and Health Research (NECRBHR) . [cited 2019 Nov 1]. Available from: https://naitik.gov.in/DHR/Homepage

4. Indian Council of Medical Research. National Ethical Guidelines for Biomedical and Health Research Involving Human participants. New Delhi: ICMR; 2017. [cited 2018 Apr 20]. Available from: http:// www.icmr.nic.in/guidelines/ICMR_Ethical_Guidelines_2017.pdf

5. Ghosh S, Shetty RS, Pattanshetty SM, Mallya SD, Pandey D, Kabekkodu $\mathrm{SP}$, et al. Human papilloma and other DNA virus infections of the cervix: A population based comparative study among tribal and general population in India. PLoS One. 2019 Jun 27;14(6):e0219173.

6. Kuyare SS, Marathe PA, Shetty YC, Kamat SK, Katkar J V, Thatte UM. Projects not initiated by investigators: a retrospective analysis of the queries raised by the institutional ethics committees of a teaching hospital. J Postgrad Med. 2014; 60(1):46-50.

7. Jalgaonkar S V, Bhide SS, Tripathi RK, Shetty YC, Marathe PA, Katkar J, et al. An audit of protocol deviations submitted to an institutional ethics committee of a tertiary care hospital. PLoS One. 2016 Jan 6;11(1):e0146334.Doi: https://doi.org/10.1371/journal.pone.0146334

8. Tripathi RK, Marathe PA, Kapse S V, Shetty YC, Kamat SK, Thatte UM. Serious adverse events reports: Analysis and outcome of review by an institutional ethics committee of a tertiary care hospital in Mumbai, India. J Empir Res Hum Res Ethics. 2016;11(3):267-73.

\section{Opposition to Haryana government's medical education directive: not just about the fee hike}

MOHIT P GANDHI

\begin{abstract}
A recent directive of the Haryana government has linked the MBBS course offered by the state government medical colleges to an annual bond of $R s$ 10 lakh. This move has been opposed by medical aspirants, medical students, as well as the medical association as it will further reduce avenues for affordable medical education. While this is indeed a grave concern, there is more that needs to be considered.
\end{abstract}

Keywords: Doctor shortage, service bond, social orientation, medical pedagogy, primary healthcare

The state government of Haryana has recently revised the fee structure for the MBBS course in its government medical colleges (1). A student will now have to execute a bond of Rs 10 lakh every year for the four-and-a-half-year course, which adds up to a minimum of Rs. 45 lakhs. This includes the annual tuition fee of Rs. 80,000 . The students may decide to pay the amount out-of-pocket each year and be free to do whatever they want after finishing the course ("Option B"). Or, they may commit to joining state government service after graduation. In this case, the State will help them get a loan

Author: Mohit P Gandhi (mohit.p.gandhi@gmail.com), Public Health Professional, 25, Mahesh Nagar Extension B, Jaipur, Rajasthan, 302015 INDIA.

To cite: Gandhi MP. Opposition to Haryana government's medical education directive: not just about the fee hike. Indian J Med Ethics. 2021 Oct-Dec; 6(4) NS: 326-28. DOI: 10.20529/IJME.2021.037.

Published online first on May 14, 2021

(c) Indian Journal of Medical Ethics 2021 while they are studying; and will start repaying it on their behalf once they join the service ("Option A"). It will take seven years for the loan amount to be fully repaid. In case the candidate wishes to leave the service at any time within the seven-year period, s/he may settle the balance amount out-of pocket and leave. The directive also allows students to switch from Option B to Option A at the beginning of any of the academic years of the course.

Before this directive, the fee was around Rs. 50,000/year, and there was no bond. By this directive, the government claims to incentivise doctors to opt for government service and to strengthen medical facilities in the State. However, "This policy does not make it mandatory/obligatory for the State Government to provide employment to the MBBS graduate" (1).

\section{Why does this need to be opposed?}

Almost half of the 542 medical colleges (MCs) in India are under non-government ownership (2). The fees in most of these colleges, even if the initial capitation is ignored, are beyond the reach of even the middle class. If the government MCs also hike their fees on such an astronomical scale, access to medical education (ME) will get limited only to the rich elite.

"Option A" appears to be a way out for those who cannot afford to pay this amount. Under this option, the State government will repay the loans of those students who join the government service after graduation. But, strangely enough, the State government has absolved itself outright of the responsibility to provide jobs to these graduates. Thus, one wonders if this is just a ploy to promote private MCs. If those colleges bring down their fees to below Rs 10 lakhs per year, a 